\title{
Multifactorial audit of invasive cervical cancer: key lessons for the National Screening Programme
}

\author{
D N Slater
}

\begin{abstract}
Aims-To audit factors associated with the development of invasive cervical cancer. Methods-Twenty cases of invasive cervical cancer in one health district for 1991-93 were audited by multifactorial analysis.

Results-The average age was 53 years with $20 \%(4 / 20)$ aged over 65 years. Of the patients, $45 \%(9 / 20)$ were identified by a cervical smear, with $40 \%(8 / 20)$ from the National Screening Programme (NSP) and $5 \%(1 / 20)$ opportunistically; $30 \%(6 / 20)$ had not received a smear, $10 \%(2 / 20)$ being aged under 65 and $20 \% 65$ or over. Those with no smear had all been hospital patients during the previous five years. No response to a smear invitation occurred in $5 \%$. In $20 \%$, there had been a true negative smear two to five years previously. Inappropriate laboratory diagnosis or inappropriate clinical management occurred in $30 \%$ and $15 \%$ (3/20), respectively. In $20 \%$, two or more factors were present in the same patient. Conclusions-Why cervical cancer occurs after a true negative smear requires research and women aged over 65 with no smear must be targeted. Failsafe systems should incorporate inadequate smears and smear adequacy should be given priority in quality assurance (QA) and training. False negative reports must be minimised but accepted as an inherent part of the NSP and not an automatic indicator of poor laboratory performance. Comprehensive national QA standards are required, to which providers must conform. Postcoital bleeding is an absolute indication for a smear and, when appropriate, opportunistic smears offered at all hospital attendances. Cervical cancer audit must be guaranteed access to all clinical and laboratory information and be seen as a means to improve the effectiveness and quality of the NSP.

(f Clin Pathol 1995;48:405-407)
\end{abstract}

Keywords: Audit, screening, cervical cancer.

Histopathology and

Cytopathology,

Rotherham NHS

Hospitals Trust,

Moorgate Road,

Rotherham S60 2UD

D N Slater

Correspondence to:

D N Slater.

Accepted for publication

13 October 1994
The audit of factors associated with the development of invasive cervical cancer is one method to assess the effectiveness of the National Screening Programme (NSP). If the Health of the Nation target is to be achieved (20\% reduction in invasive cervical cancer by the year 2000), it is probable that contributory factors revealed by audit will require rectification. Information on factors associated with the development of cervical cancer has been largely derived from a small number of studies, each of which tended to analyse one specific aspect. ${ }^{1-6}$ A pilot audit of cervical screening histories has been undertaken jointly by the National Co-ordinating Network and Imperial Cancer Research Fund, although the results are not yet published and the audit did not undertake smear review. Few published studies have undertaken a comprehensive multifactorial audit and assessed the relative frequency of different factors. ${ }^{78}$ However, one relevant to the current study was based on deaths from cervical cancer in Rotherham district for the period $1989-91 .^{8}$ It has been suggested that a confidential national enquiry into cervical cancer should be undertaken, although a mandatory NSP directive to audit this area is still awaited. Also, little attention has been paid to the impact of the NSP on factors associated with the development of invasive cervical cancer. For these reasons, a multifactorial audit of invasive cervical cancer was undertaken in Rotherham district for the period 1991-93. This period represented five to eight years after a computed NSP had been introduced, based on a five year recall interval. The results permitted comparison with the earlier audit of deaths from cervical cancer in the same district.

\section{Methods}

Details of invasive cervical cancer in Rotherham district for the period 1991-93 were obtained from the computerised records of the Rotherham Hospital Cytopathology Department. A multifactorial audit was performed by a review of laboratory and hospital records, together with, when appropriate, review of general practitioner records and re-screening of cytology smears. Factors associated with the development of invasive cervical cancer were classified under the headings shown in the table. There was no overlap of cases between this and the previously published audit of deaths from cervical cancer. The information obtained did not relate purely to Rotherham Hospitals NHS Trust or Rotherham general practitioners. In $40 \%$ of cases, previous clinical and/or laboratory information was obtained from either NHS hospitals outside the district or non-NHS sources. All hospital laboratories 
Presentation and factors associated with incident invasive cervical cancer in 20 cases presenting between 1991 and 1993

\begin{tabular}{lr}
\hline & Total (\%) \\
\hline $\begin{array}{l}\text { Presentation } \\
\text { NSP }\end{array}$ & $8(40)$ \\
opportunistic smear & $1(5)$ \\
symptomatic & $11(55)$ \\
Single factors & \\
no previous cytology record & $6(30)$ \\
total & $2(10)$ \\
under 65 years (pre-call) & $4(20)$ \\
over 65 years & $1(5)$ \\
no response to invitation & $4(20)$ \\
true negative smear & $16(30)$ \\
inappropriate laboratory report & $2(10)$ \\
total & $7(20)$ \\
undergraded & $2(10)$ \\
false negative (total) & $4(10)$ \\
missed inadequate & $6(30)$ \\
missed dyskaryosis & $3(15)$ \\
no hospital opportunistic smear & $0(0)$ \\
inappropriate clinical management & $4(20)$ \\
problems in failsafe & \\
Two or more factors &
\end{tabular}

involved had performed satisfactorily in the national gynaecological cytology proficiency testing scheme.

\section{Results}

Twenty cases of invasive cervican cancer were identified. The average age was 53 years with $20 \%(4 / 20)$ being over 65 years of age. The mode of presentation was symptomatic in 55\% $(11 / 20)$ and as a result of a cervical smear in $45 \%(9 / 20)$. Of the latter, $40 \%(8 / 20)$ were NSP smears and the remaining $5 \%(1 / 20)$ opportunistic smears. Symptomatic presentations in those under 65 years of age all resulted from postcoital bleeding. Of the cases, $30 \%(6 / 20)$ had no record of a previous cervical smear invitation, $10 \%(2 / 20)$ of whom were under 65 years of age and $20 \% 65$ years or over. All those under 65 years of age had presented before they had been called. No response to a NSP cervical smear invitation was identified in only $5 \%$. All individuals with no record of a cervical smear had been hospital inpatients, for non-gynaecological reasons, at least once during the previous five years. In addition, one third of these had also received hospital gynaecological consultations for family planning or pregnancy related problems. However, in no instance had an opportunistic smear been undertaken.

In $20 \%$ of cases (average age 36 years, range 29-46 years) a true negative smear had been reported between two and five years previously (average 4.2 years). An inappropriate laboratory diagnosis was identified in $30 \%$ of cases, $20 \%$ of which represented false negative reports, and included $10 \%$ of cases where an inadequate smear had been reported as negative. The cases of missed dyskaryosis fell into the "few cells and easily missed category", as defined by Robertson. 9 In two cases an inappropriate laboratory diagnosis had occurred on two or more occasions. Inappropriate clinical management was identified in $15 \%$ $(3 / 20)$ of cases. Specific examples included up to two years to repeat an inadequate smear and two years to investigate postmenopausal bleeding. In $20 \%$ of cases two or more different factors were identified in the same patient.

\section{Discussion}

In $40 \%$ of cases, invasive cervical cancer had been identified by the NSP. This lends support to the view that the NSP is having increasing effect.

Factors associated with invasive cervical cancer fell into easily definable groups, with frequencies between 15 and $30 \%$. Some of these groups were identical with those identified in the previous study of cervical cancer deaths. ${ }^{8}$ These included women over 65 years of age with no cytology record, true negative smears, laboratory errors, and inappropriate clinical management. However, in contrast to the previous study, there was a considerably smaller percentage of non-responders to a cervical smear invitation. There was also a fall in the percentage of women under 65 years of age with no cytology record, although the latter had been predicted as a consequence of the call programme. ${ }^{8}$ As in the previous study, there was no example of an abnormal smear being followed up inappropriately although, paradoxically, no district involved in the study had completed implementation of a laboratory based failsafe system. Also, in common with the previous study, $20 \%$ of patients had contributory factors belonging to at least two groups.

A new group identified by the present study included those women who presented symptomatically with no record of a previous cervical smear. For various reasons, all had been hospital patients at least once during the previous five years. However, an opportunistic smear test had not been undertaken on any occasion. Hospital in- and outpatients comprise a large female population. Accordingly, it is recommended that, at all hospital attendances, details of past medical history should routinely include cervical smear history. Then, if indicated, an opportunistic smear test could be advised. In addition, it is significant that all symptomatic presentations occurring in those under 65 years of age were due to postcoital bleeding, which must be highlighted as an absolute clinical indication for a cervical smear.

As in the previous study, the percentage of cases associated with a previous true negative smear is of concern and provides further support for a three rather than a five year recall interval. Furthermore, the contribution of poor smear taking to cytologically true negative smears requires further investigation.

Cervical smear inadequacy was associated with both clinical and laboratory problems in $20 \%$ of cases. In $5 \%$, inadequate smears had taken up to two years to be repeated. Current national guidelines for failsafe mechanisms do not incorporate inadequate smears, ${ }^{1011}$ although this study indicates the clear necessity to do so. In $10 \%$ of cases misreported inadequate smears were the cause of a false negative laboratory report. Despite a national statement on smear adequacy, ${ }^{12}$ there is still substantial interdistrict variation in the reporting frequency of inadequate smears. For these reasons, it is essential there is continuing education and research into the definition of smear adequacy, supported by both audit and inclusion of in- 
adequate smears in external quality assessment schemes. The latter would appear particularly pertinent as there have been suggestions that inadequate smears be removed from the national proficiency testing scheme.

Missed dyskaryosis was the cause of a false negative report in $10 \%$ of cases and all attempts must be made to minimise laboratory errors. However, there must be wider education that false negative reports, although unfortunate, are an inherent component of any screening programme. There is no national guidance as to what constitutes an acceptable laboratory false negative rate. Accordingly, there would appear to be an urgent requirement to set such standards for quality assurance, to which laboratory performance must conform. ${ }^{1314}$

In $40 \%$ of cases the previous clinical or laboratory information was obtained from either NHS hospitals and general practitioners outside the district or non-NHS sources. However, for audit into invasive cervical cancer to be successful, full access to all relevant clinical and laboratory information must be guaranteed. This may require a national directive to ensure its confidential release. Also, to be comprehensive, audit into invasive cervical cancer must include smear review.

A proposed essential component of the NSP is the multifactorial audit of both invasive cervical cancer and deaths from cervical cancer. ${ }^{8}$
The lessons learnt are the key to improving the effectiveness and quality of the NSP.

1 Fletcher A. Screening for cancer of the cervix in elderly women. Lancet 1990;335:97-9.

2 Mitchell H, Medley G, Giles G. Cervical cancers diagnosed after negative results on cervical cytology: perspective in the 1980's. BMF 1990;300:1622-4.

3 Nathoo V. Invitation of non-responders at a cervical cancer screening clinic in Manchester. BMF 1988;296:1041-2.

4 Wilson SH, Johnson J. An audit of cervical cancer deaths in Nottingham. Cytopathology 1992;3:79-84.

5 Patterson MEL, Peel AR, Joslin CAF. Cervical smear histories of 500 women with invasive cervical cancer in Yorkshire. BMF 1984;289:896-8.

6 Elwood JM, Cotton RE, Johnson J, Jones GM, Curnow J, Beaver MW. Are patients with abnormal cervical smears adequately managed? $B M \mathcal{F}$ 1984;289:891-4.

7 Hunt CM, Lamb NP, Breeson AJ, Vellacott ID, Devonald E, Purnell C, et al. Cervical screening: checking effectiveness by auditing 'system failures'. Cytopathology 1993; 4(Suppl 1):5.

8 Slater DN, Milner PC, Radley H. Audit of deaths from cervical cancer: Proposal for an essential component of the National Screening Programme. $\mathcal{f}$ Clin Pathol 1994; 47:27-8. 9 Robertson JH, Woodend B. Negative cytology preceding

10 Pike C, Chamberlain J. Guidelines on Fail-Safe Actions. Oxford: National Co-Ordinating Network Publication, 1992:1-12.

11 NHS Management Executive. Failsafe mechanisms for the follow-up of cervical smears. EL(92)39. London: Department of Health, 1992 .

12 Statement by the British Society for Clinical Cytology and the British Society for Colposcopy and Cervical Cytology. Cell content of cervical smears. Cytopathology 1990;1: 129-30.

13 Slater DN. Cervical cytology internal quality assurancewhat are the national standards? Cytopathology 1994;5: 207-10. 14 Slater DN. Cervical cytology external and internal quality
assurance: a comparative appraisal. $f$ Clin Pathol 1995; 